\title{
FACTORIZED VARIATIONAL APPROXIMATIONS FOR ACOUSTIC MULTI SOURCE LOCALIZATION
}

\author{
V. Cevher*, A. C. Sankaranarayanan, and R. Chellappa \\ Center for Automation Research, \\ University of Maryland, \\ College Park, MD 20742 \\ \{volkan, aswch,rama\}@cfar.umd.edu
}

\begin{abstract}
Estimation based on received signal strength (RSS) is crucial in sensor networks for sensor localization, target tracking, etc. In this paper, we present a Gaussian approximation of the Chi distribution that is applicable to general RSS source localization problems in sensor networks. Using our Gaussian approximation, we provide a factorized variational Bayes (VB) approximation to the location and power posterior of multiple sources using a sensor network. When the source signal and the sensor noise have uncorrelated Gaussian distributions, we demonstrate that the envelope of the sensor output can be accurately modeled with a multiplicative Gaussian noise model. In turn, our factorized VB approximations decrease the computational complexity and provide computational robustness as the number of targets increases. Simulations are provided to demonstrate the effectiveness of the proposed approximations.
\end{abstract}

Index Terms - Variational Methods, Stochastic Approximation, Multisensor Systems, Object Tracking

\section{INTRODUCTION}

Detection and localization based on received signal strength (RSS) are important problems for sensor networks and pose interesting challenges for statistical signal processing. The objective in these problems is the detection, localization, and tracking of objects based on the received power measurements at spatially distributed sensors. As the power of the signal is easy to compute and does not require high bandwidth or accurate synchronization to transmit, estimation based on RSS has become quite popular in sensor networks [1].

In this paper, we derive a factorized variational Bayes (VB) approximation to the joint posterior of power and location of multiple acoustic sources for efficient inference [2-4]. When the number of targets is $K$, there are $3 \times K$ unknown parameters that need to be jointly solved using the RSS measurements from multiple sensors: one parameter for each source power and two parameters for each source location over a planar area. A direct solution results in a computational complexity, which is exponential in $K$. Our factorized VB approximation results in a computational complexity, which is linear with $K$. Note that for this problem, the posterior density is not directly factorizable. We provide an approximation that decouples the power estimation and localization of multiple sources, which alleviates the tractability.

We assume that the source signals appear as noisy sinusoids over short time segments and provide a Gaussian approximation of the Chi

Prepared through collaborative participation in the Advanced Sensors Consortium sponsored by the U. S. Army Research Laboratory under the Collaborative Technology Alliance Program, Cooperative Agreement DAAD19-01-02-0008.

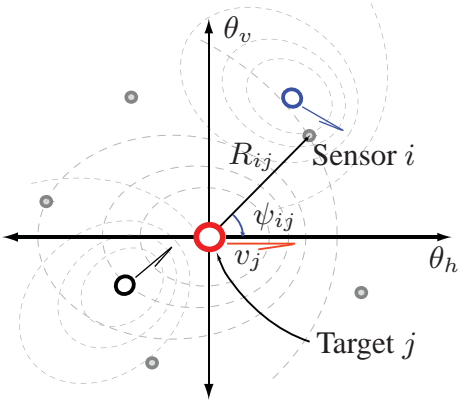

Fig. 1. This figure illustrates the geometry of the sensor-target configuration for multiple monopole acoustic sources moving on the $\theta_{h}-\theta_{v}$ plane. The dashed lines are the acoustic wave-fronts, which create the interaction between the sources and the sensors.

distribution, which appears when the power of Gaussian sources is calculated. Our approximation is based on moment matching and is quite accurate even at moderate sample sizes [2]. Most signals with a harmonic frequency structure satisfy our source assumption: e.g., motor vehicles. In this paper, we focus on the localization of the sources. In [2], we also derive the Cramer-Rao lower bound for the localization parameter and the receiver operating characteristics for target detection.

The organization of the paper is as follows. Section 2 introduces the signal model and describes our Gaussian approximation of the Chi distribution. Section 3 applies the variational Bayes approach to determine a factorized approximation to the multi target power and location posterior. Computer simulations are provided in Section 4 to demonstrate the estimation performance of the proposed approximations.

\section{SIGNAL MODEL AND DENSITY APPROXIMATIONS}

We discuss the location estimation of $K$-narrow-band sources with an acoustic sensor network consisting of $M$-omnidirectional single microphone sensors in an isotropic medium. We assume that there are no multipath effects. We denote $s_{j}(t), x_{i j}(t), n_{i}(t)$, and $y_{i}(t)$ as the complex envelopes of the $j$ th source signal $(j=1, \ldots, K$, where $K$ is assumed to be known), the $j$ th source signal at the $i$ th sensor $(i=1, \ldots, M$, where $M$ is assumed to be known), the $i$ th sensor additive noise, and the $i$ th sensor output signal, respectively. The $j$ th source position is denoted as $\boldsymbol{\theta}_{j}=\left[\begin{array}{ll}\theta_{h, j}, & \theta_{v, j}\end{array}\right]^{\prime}$ and the position of the $i$ th sensor as $\boldsymbol{\zeta}_{i}=\left[\begin{array}{ll}\zeta_{h, i}, & \zeta_{v, i}\end{array}\right]^{\prime}$. Assuming a propagation loss constant $\alpha$, we 

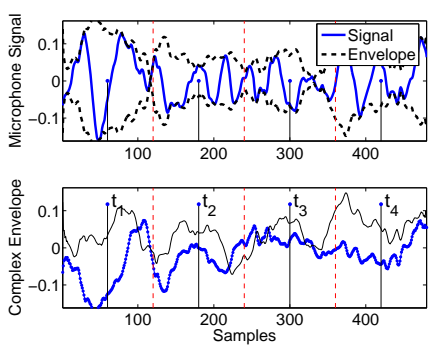

Fig. 2. (Top) A short segment of a Volkswagen Passat drive-by noise is shown where $F_{s}=48 \mathrm{kHz}$. A total of 480 samples are divided into $N=4$ segments, where each segment indexed by $t_{i}(i=1, \ldots, 4)$ has 120 samples. As illustrated by the top plot, the observed signal at each segment looks like a noisy sinusoid, whose dominant frequency is approximately $1100 \mathrm{~Hz}$. (Bottom) Real (solid) and imaginary (circles) parts of the complex envelope are illustrated.

write the complex envelope of the $i$ th sensor output signal at the narrowband frequency $f_{0}$ of the sources using the superposition principle $[5,6]$ (see Figs. 1 and 2):

$$
y_{i}(t)=\sum_{j=1}^{K} x_{i j}(t)+n_{i}(t)=\sum_{j=1}^{K} \frac{s_{j}(t)}{\sqrt{\beta_{i j}} R_{i j}^{\alpha / 2}} e^{-j \frac{2 \pi f_{0} R_{i j}}{\beta_{i j} c}}+n_{i}(t),
$$

where $R_{i j}$ is the range of the $j$ th source to the $i$ th sensor, $v_{j}$ is the $j$ th source speed, $\psi_{i j}$ is the angle of the $j$ th source speed vector with respect to the line connecting the $j$ th source and the $i$ th sensor, $\beta_{i j}=$ $1+\frac{v_{j}}{c} \cos \psi_{i j}$ is the Doppler shift factor, and $c$ is the speed of sound.

To calculate the RSS at a sensor, $N$ snapshots of the acoustic envelope, calculated at times $\left(t_{1}, \ldots, t_{N}\right)$, are used (see Fig. 2 ). We note that if the time samples are sufficiently apart, then successive samples of the source and the noise samples are uncorrelated [7]. We model the $j$ th source signal as i.i.d., zero mean, complex circularly symmetric Gaussian random variables $\mathcal{C N}\left(0, \sigma_{j}^{2}\right)$ with variance $\sigma_{j}^{2}$ and the noise samples $n_{i}(t) \sim \mathcal{C N}\left(0, \sigma^{2}\right)$ with variance $\sigma^{2}$.

Based on our signal and noise assumptions, it is straightforward to prove that the $i$ th sensor output signal $y_{i}(t)$ also has an i.i.d. zero mean circularly complex Gaussian distribution with variance $\sigma_{y, i}^{2}=\sum_{j} \sigma_{x, i j}^{2}+$ $\sigma_{i}^{2}$, where $\sigma_{x, i j}^{2}=\frac{\sigma_{j}^{2}}{\beta_{i j} R_{i j}^{\alpha}}$. Now, we denote the $N$-sample RSS output of the $i$ th sensor as $\varepsilon_{i}=\sqrt{\frac{1}{N} \sum_{n=1}^{N}\left|y_{i}\left(t_{n}\right)\right|^{2}}$ :

$$
\varepsilon_{i}=\frac{\sigma_{y, i}}{\sqrt{2 N}} \sqrt{\sum_{n=1}^{N}\left(\frac{y_{i, \text { real }}^{2}\left(t_{n}\right)}{\sigma_{y, i}^{2} / 2}+\frac{y_{i, \mathrm{imag}}^{2}\left(t_{n}\right)}{\sigma_{y, i}^{2} / 2}\right)}=\frac{\sigma_{y, i}}{\sqrt{2 N}} z,
$$

where we define $z$ as the second square-root summation term in (2). The variable $Z$ has a Chi distribution $p_{Z}(\cdot)$ with $2 N$ DOF [8]. Then, $\varepsilon_{i}$ has the following distribution: $\varepsilon_{i} \sim \frac{\sqrt{2 N}}{\sigma_{y, i}} p_{Z}\left(\frac{\sqrt{2 N}}{\sigma_{y, i}} \varepsilon_{i}\right)$. In [2], we provide Gaussian approximations for the Chi distribution, which are accurate even at moderate sample sizes. Using moment matching [2], the RSS distribution can be approximated by

$$
\varepsilon_{i} \approx p\left(\varepsilon_{i}\right)=\mathcal{N}\left(\sqrt{\frac{2 N-1}{2 N}} \sigma_{y, i}, \frac{\sigma_{y, i}^{2}}{4 N}\right) \approx \sigma_{y, i} e^{\frac{\mathcal{N}(0,1)}{\sqrt{4 N}}},
$$

where we used $\sqrt{\frac{2 N-1}{2 N}} \approx 1$ for $N \gg 1$ and $e^{x} \approx 1+x$ for $x \ll 1$. Hence, we approximate the acoustic RSS output with a multiplicative noise model. In [2], we compare this approximation with other approximations of the acoustic RSS output typically found in the literature and

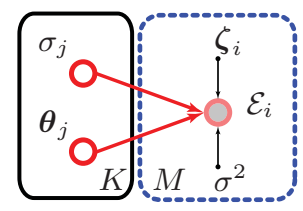

Fig. 3. A directed acyclic graph is used to represent the inference problem, where the dashed box denotes the set of $M$ acoustic sensor observations $\mathcal{E}_{i}$, which are assumed independent. The deterministic (known) components of the problem are shown with solid dots. The shaded node represents the observed variables $\mathcal{E}_{i}$, whereas the remaining nodes in the solid box represents the $K$-target latent variables $\sigma_{j}$ and $\boldsymbol{\theta}_{j}$. Note that $\sigma_{j}$ and $\boldsymbol{\theta}_{j}$ 's are independent. However, given $\mathcal{E}_{i}$ 's, they are entangled.

show that this approximation results in smaller range estimation bias and better error estimates.

\section{FACTORIZED POSTERIOR APPROXIMATIONS}

In the acoustic target localization problem, the objective of the sensor network is to determine the $K$ target locations $\boldsymbol{\theta}_{j}$ 's, which are entangled with the source signal powers $\sigma_{j}^{2}$ 's via (1). In this section, we address the joint estimation of these latent variables, which is summarized as a graphical model in Fig. 3. Instead of using the complex envelope samples $\left\{s_{i}(t)\right\}_{t_{1}}^{t_{N}}$, we only use the sufficient statistics $\mathcal{E}_{i} \triangleq \log \varepsilon_{i}$ from each sensor $i$, which can completely determine the distribution of $s_{i}(t)$ given $\sigma_{j}^{2}$ 's and $\boldsymbol{\theta}_{j}$ 's. We assume that the propagation loss constant is $\alpha=2$ and the targets have moderate speeds so that the effects of the Doppler factors can be ignored. To increase the tractability of the solution for the latent variables $\boldsymbol{Z}=\left[\boldsymbol{\sigma}, \boldsymbol{\Theta}^{\prime}\right]^{\prime}\left(\boldsymbol{\sigma}=\left[\sigma_{1}, \ldots, \sigma_{K}\right], \boldsymbol{\Theta}=\left[\boldsymbol{\theta}_{1}^{\prime}, \ldots, \boldsymbol{\theta}_{K}^{\prime}\right]^{\prime}\right)$ from the observations $\mathcal{E}=\left[\mathcal{E}_{1}, \ldots, \mathcal{E}_{M}\right]$, we propose using a factorized approximation to the joint posterior distribution of these variables as $p(\boldsymbol{Z}, \mathcal{E})=\prod_{j=1}^{K} f_{\sigma_{j}}\left(\sigma_{j}\right) f_{\boldsymbol{\theta}_{j}}\left(\boldsymbol{\theta}_{j}\right)$. The construction of our approximation follows the factorized variational Bayesian (VB) approximations commonly used in the literature $[3,4]$.

Under the mean field theory framework, we consider minimizing the Kullback-Leibler $(\mathrm{KL})$ divergence of the full posterior $p(\boldsymbol{Z}, \mathcal{E})$ from our factorized approximation $\prod_{j=1}^{K} f_{\sigma_{j}}\left(\sigma_{j}\right) f_{\boldsymbol{\theta}_{j}}\left(\boldsymbol{\theta}_{j}\right)[3,4]$. Then, the optimal factors $f^{*}$,s can be determined from the following implicit expressions:

$$
\begin{aligned}
& f_{\sigma_{j}}^{*}\left(\sigma_{j}\right) \propto \exp \left(E_{\boldsymbol{\Theta}} E_{\boldsymbol{\sigma} \backslash \sigma_{j}} \log p(\boldsymbol{Z}, \mathcal{E})\right), \\
& f_{\boldsymbol{\theta}_{j}}^{*}\left(\boldsymbol{\theta}_{j}\right) \propto \exp \left(E_{\boldsymbol{\sigma}} E_{\boldsymbol{\Theta} \backslash \boldsymbol{\theta}_{j}} \log p(\boldsymbol{Z}, \mathcal{E})\right) .
\end{aligned}
$$

Note that (4) does not represent an explicit solution as the solutions of the optimal factors depend on expectations computed with respect to other factors. Therefore, (4) denotes a set of consistency conditions, which can be cycled to reach a consensus. It can be shown that convergence is guaranteed $[3,4]$.

From the graphical model in Fig. 3, the posterior density factors as $p(\boldsymbol{Z}, \mathcal{E})=\prod_{j=1}^{K} p\left(\sigma_{j}\right) p\left(\boldsymbol{\theta}_{j}\right) \prod_{i=1}^{M} p\left(\mathcal{E}_{i} \mid \boldsymbol{Z}\right)$, where, due to our Gaussian approximation in $(3), p\left(\mathcal{E}_{i} \mid \boldsymbol{Z}\right) \approx \mathcal{N}\left(\log \sigma_{y, i}, \frac{1}{4 N}\right)$, and $p\left(\sigma_{j}\right)$ and $p\left(\boldsymbol{\theta}_{j}\right)$ are prior distributions for $\sigma_{j}$ and $\boldsymbol{\theta}_{j}$. We restrict the form of the posterior factors (4) as follows:

$$
f_{\sigma_{j}}^{*}\left(\sigma_{j}\right) \sim \log -\mathcal{N}\left(\mu_{j}, v_{j}^{2}\right) ; f_{\boldsymbol{\theta}_{j}}^{*}\left(\boldsymbol{\theta}_{j}\right) \sim \mathcal{N}\left(\boldsymbol{\theta}_{j}, \boldsymbol{\Sigma}_{j}\right),
$$

and we use the following conjugate prior distributions:

$$
p\left(\sigma_{j}\right) \sim \log -\mathcal{N}\left(\mu_{j, 0}, v_{j, 0}^{2}\right) ; p\left(\boldsymbol{\theta}_{j}\right) \sim \mathcal{N}\left(\boldsymbol{\theta}_{j, 0}, \boldsymbol{\Sigma}_{j, 0}\right) .
$$


We emphasize that the posterior density does not factorize directly in terms of $\sigma_{j}$ and $\boldsymbol{\theta}_{j}$ :

$$
\begin{aligned}
\log p(\boldsymbol{Z}, \mathcal{E}) & =-2 N \sum_{i=1}^{M}\left[\mathcal{E}_{i}-\frac{1}{2} \log \left(\sum_{j=1}^{K} \frac{\sigma_{j}^{2}}{R_{i j}^{2}}+\sigma^{2}\right)\right]^{2} \\
- & \sum_{j=1}^{K}\left[\frac{\left(\log \sigma_{j}-\mu_{j, 0}\right)^{2}}{2 v_{j, 0}^{2}}+\log \sigma_{j}\right]-\frac{1}{2}\left(\boldsymbol{\Theta}-\boldsymbol{\Theta}_{0}\right)^{\prime} \boldsymbol{\Sigma}_{0}^{-1}\left(\boldsymbol{\Theta}-\boldsymbol{\Theta}_{0}\right),
\end{aligned}
$$

where $\boldsymbol{\Sigma}_{0}=\operatorname{diag}\left\{\boldsymbol{\Sigma}_{1,0}, \ldots, \boldsymbol{\Sigma}_{K, 0}\right\}$.

To proceed further, we perform a Taylor series expansion of the $\log \sigma_{y, i}^{2}$ term in the posterior density (7):

$$
\begin{aligned}
\log \sigma_{y, i}^{2}=\log \left(\sum_{j=1}^{K} \frac{\sigma_{j}^{2}}{R_{i j}^{2}}+\sigma^{2}\right) \approx \kappa_{i, 0}+\sum_{j=1}^{K} \lambda_{i j, 0} \log \frac{\sigma_{j}^{2}}{R_{i j}^{2}}, \text { where } \\
\lambda_{i j, 0}=\frac{e^{\left(2 \mu_{j, 0}\right)} / R_{i j, 0}^{2}}{\sum_{k=1}^{K}\left(e^{\left(2 \mu_{k, 0}\right)} / R_{i k, 0}^{2}\right)}, \\
\kappa_{i, 0}=\log \left(\sum_{j=1}^{K} \frac{e^{\left(2 \mu_{j, 0}\right)}}{R_{i j, 0}^{2}}+\sigma^{2}\right)-\sum_{j=1}^{K} \lambda_{i j, 0} \log \frac{e^{\left(2 \mu_{j, 0}\right)}}{R_{i j, 0}^{2}},
\end{aligned}
$$

and $R_{i j, 0}=\left|\boldsymbol{\theta}_{j, 0}-\boldsymbol{\zeta}_{i}\right|$. The expansion in (8) enables a VB factorization of the posterior (4), which is detailed below.

\subsection{VB Cycle on Source Powers}

To update the $j$ th source's power, we take the expected value of the logarithm of the posterior density (7) with respect to the all the source positions $\Theta$ and the all the other source powers $\sigma \backslash \sigma_{j}$. We define $\hat{\mathcal{E}}_{i, 0}=\mathcal{E}_{i}-0.5 \kappa_{i, 0}$ and approximate $E_{\Theta} \log R_{i j} \approx \log R_{i j, 0}$. Then, by substituting (8) into (7), we determine the required expectation, which has a log-Normal form:

$$
\begin{aligned}
\log f_{\sigma_{j}}^{*}\left(\sigma_{j}\right) \doteq-\sum_{j=1}^{K}\left[\frac{\left(\log \sigma_{j}-\mu_{j, M}\right)^{2}}{2 v_{j, M}^{2}}+\log \sigma_{j}\right] \text {, where } \\
\frac{1}{v_{j, M}^{2}}=4 N \sum_{i=1}^{M} \lambda_{i j, 0}^{2}+\frac{1}{v_{j, 0}^{2}}, \\
\frac{\mu_{j, M}}{v_{j, M}^{2}}=4 N \sum_{i=1}^{M} \hat{\mathcal{E}}_{i, 0} \lambda_{i j, 0}-4 N \sum_{i=1}^{M} \sum_{\substack{k=1 \\
k \neq j}}^{K} \lambda_{i j, 0} \lambda_{i k, 0} \mu_{k, 0} \\
\quad+2 N \sum_{i=1}^{M} \sum_{k=1}^{K} \lambda_{i j, 0} \lambda_{i k, 0} \log R_{i j, 0}+\frac{\mu_{j, 0}}{v_{j, 0}^{2}} .
\end{aligned}
$$

The derivation is omitted due to lack of space.

\subsection{VB Cycle on Source Locations}

To update the $j$ th source's location, we take the expected value of the logarithm of the posterior density (7) with respect to the all the other source positions $\Theta \backslash \boldsymbol{\theta}_{j}$ and the all source powers $\boldsymbol{\sigma}$. We define $\mathfrak{E}_{i j, 0}=$ $\hat{\mathcal{E}}_{i, 0}-\sum_{k=0}^{K} \lambda_{i k, 0} \mu_{k, 0}+\sum_{k=1, k \neq j}^{K} \lambda_{i k, 0} \log R_{i j, 0}$. Then, similar to the VB cycle on source powers, we take the expected value of the logarithm of the posterior (7) to obtain:

$$
\begin{aligned}
\log f_{\boldsymbol{\theta}_{j}}^{*}\left(\boldsymbol{\theta}_{j}\right) \doteq-2 N \sum_{i=1}^{M} & {\left[\mathfrak{E}_{i j, 0}+\lambda_{i j, 0} \log R_{i j}\right]^{2} } \\
& -\frac{1}{2}\left(\boldsymbol{\theta}_{j}-\boldsymbol{\theta}_{j, 0}\right)^{\prime} \boldsymbol{\Sigma}_{j, 0}^{-1}\left(\boldsymbol{\theta}_{j}-\boldsymbol{\theta}_{j, 0}\right) .
\end{aligned}
$$

Input: Prior density parameters: $\left\{\mu_{j, 0}, v_{j, 0}, \boldsymbol{\theta}_{j, 0}, \boldsymbol{\Sigma}_{j, 0}\right\}_{j=1}^{K}$ and the sensor network data $\mathcal{E}$.

Output: Factorized posterior density parameters for approximate Iterations $=0$; repeat $\{\mathrm{VB}$ Cycles

Linearize $\log \sigma_{y, i}^{2}$ using (8) to obtain $\kappa_{i, 0} \lambda_{i j, 0}$ (9);

$\hat{\mathcal{E}}_{i, 0}=\mathcal{E}_{i}-0.5 \kappa_{i, 0}$;

$\mathfrak{E}_{i j, 0}=\hat{\mathcal{E}}_{i, 0}-\sum_{k=0}^{K} \lambda_{i k, 0} \mu_{k, 0}+\sum_{k=1, k \neq j}^{K} \lambda_{i k, 0} \log R_{i j, 0} ;$

foreach source power $\sigma_{j}, j \leftarrow 1$ to $K$ do

| Calculate $\mu_{j, M}(1 \times 1), v_{j, M}(1 \times 1)$ using (11);

end

foreach source location $\boldsymbol{\theta}_{j}, j \leftarrow 1$ to $K$ do

Determine the mode $\boldsymbol{g}_{j, 0}(2 \times 1)$ and the Hessian

$\boldsymbol{H}_{j, 0}(2 \times 2)$ at the mode of $\log f_{\boldsymbol{\theta}_{j}}^{*}\left(\boldsymbol{\theta}_{j}\right)(12)$;

Calculate $\boldsymbol{\theta}_{j, M}, \boldsymbol{\Sigma}_{j, M}$ using (14);

end

for $j \leftarrow 1$ to $K$ do

| $\left\{\mu_{j, 0}, v_{j, 0}, \boldsymbol{\theta}_{j, 0}, \boldsymbol{\Sigma}_{j, 0}\right\} \longleftarrow\left\{\mu_{j, M}, v_{j, M}, \boldsymbol{\theta}_{j, M}, \boldsymbol{\Sigma}_{j, M}\right\} ;$

end

Iterations $\leftarrow$ Iterations +1 ;

until Iterations $=$ VBIts;

for $j \leftarrow 1$ to $K$ do

$\mid\left\{\mu_{j}, v_{j}, \boldsymbol{\theta}_{j}, \boldsymbol{\Sigma}_{j}\right\} \longleftarrow\left\{\mu_{j, 0}, v_{j, 0}, \boldsymbol{\theta}_{j, 0}, \boldsymbol{\Sigma}_{j, 0}\right\} ;$

end

Algorithm 1: Factorized VB algorithm for multiple source localization using RSS.

Unfortunately, (12) cannot be rearranged in a Gaussian form. Since it requires too much computation to numerically obtain the Gaussian approximation directly from the KL divergence, we approximate this VB cycle using the Laplacian approximation [9]:

$$
\begin{gathered}
\log f_{\boldsymbol{\theta}_{j}^{*}}^{*}\left(\boldsymbol{\theta}_{j}\right) \dot{\sim}-\frac{1}{2}\left(\boldsymbol{\theta}_{j}-\boldsymbol{\theta}_{j, M}\right)^{\prime} \boldsymbol{\Sigma}_{j, M}^{-1}\left(\boldsymbol{\theta}_{j}-\boldsymbol{\theta}_{j, M}\right), \text { where } \\
\boldsymbol{\Sigma}_{j, M}^{-1}=\boldsymbol{H}_{j, 0}, \text { and } \boldsymbol{\theta}_{j, M}=\boldsymbol{g}_{j, 0} .
\end{gathered}
$$

In (14), we use (12) and numerically calculate the mode $\boldsymbol{g}_{j, 0}=\arg \max _{\boldsymbol{\theta}_{j}}$ $\log f_{\boldsymbol{\theta}_{j}^{*}}^{*}\left(\boldsymbol{\theta}_{j}\right)$ and the Hessian $\boldsymbol{H}_{j, 0}=\left.\nabla_{\boldsymbol{\theta}_{j}}^{2} \log f_{\boldsymbol{\theta}_{j}^{*}}^{*}\left(\boldsymbol{\theta}_{j}\right)\right|_{\boldsymbol{\theta}_{j}=\boldsymbol{g}_{j, 0}}$ for each source $j$. The mode $\left(\operatorname{size}\left(\boldsymbol{g}_{j, 0}\right): 2 \times 1\right)$ and the Hessian $\left(\operatorname{size}\left(\boldsymbol{H}_{j, 0}\right)\right.$ : $2 \times 2$ ) are calculated by interior point optimization methods such as Newton-Raphson recursion. Because of the prior term, the behavior of the optimization algorithm is quite stable. A pseudo code of the VB cycles is given in Algorithm 1.

\section{SIMULATIONS}

Figure 4 illustrate the results of Monte-Carlo (MC) runs that compare the factorized VB estimation algorithm with the maximum likelihood (ML) method, which jointly solves the multiple source powers and locations. Hence, the ML method maximizes the logarithm of the joint posterior in (7) without any approximations. In the simulations, a sensor network of 100 sensors is uniformly deployed on an area of radius $100 \mathrm{~m}$, which corresponds to a density of roughly one sensor per $18 \mathrm{~m}^{2}$. In the MC runs, we vary the sensor locations, the target signals according to (1), the source powers $p\left(\sigma_{j}\right)=\log -\mathcal{N}\left(80 \mathrm{~dB},(3 \mathrm{~dB})^{2}\right)$, and the initial target locations. The MC run sizes are $K \times 10^{4}$ for the different scenarios.

We provide two different configurations. In the first configuration shown in Fig. 4(Top), three closely spaced targets are localized where the 
Table 1. Performance (VB \ML)

\begin{tabular}{||c||c|c||}
\hline \hline Huber Estimator $^{1}$ & $K=3$ & $K=7$ \\
\hline \hline Amplitude Bias $^{\dagger}$ & $0.17 \backslash 1.24$ & $0.15 \backslash-0.12$ \\
\hline Amplitude Error RMS $^{\dagger}$ & $2.41 \backslash 3.1$ & $2.28 \backslash 2.36$ \\
\hline \hline Absolute Location Bias & $0.43 \backslash 2.14$ & $0.54 \backslash 0.08$ \\
\hline Location Error RMS & $8.47 \backslash 14.1$ & $7.33 \backslash 9.12$ \\
\hline \hline
\end{tabular}

* We use nearest neighbor classification in location to assign estimates to targets. We then use Huber's M-estimator to reduce the effects of outliers [10].

${ }^{\dagger}$ In units of $\mathrm{dB}$.

target locations are initialized randomly as shown in Fig. 4(a). For this simulation, we use VBIts $=30$, and the prior density on target locations is given by $p\left(\boldsymbol{\theta}_{j}\right) \sim \mathcal{N}\left(\boldsymbol{\theta}_{\text {sample }}, 20^{2} \boldsymbol{I}_{2 \times 2}\right)$ whereas the prior density of the source powers is $p\left(\sigma_{j}\right)=\log -\mathcal{N}\left(80 \mathrm{~dB},(3 \mathrm{~dB})^{2}\right)$. The results of the VB and ML algorithms are visualized in Fig. 4(b) and (c), where the target locations are shown with $\otimes$ 's and algorithm estimates are shown with 's. Figure 4(d) shows the histogram of the source power estimates of the both methods (VB: solid line, ML: dashed line) as well as the initial source power histogram (dotted line). The ML solution has problem converging to the true locations when target locations are randomly initialized as opposed to an initialization matching the prior density $p\left(\boldsymbol{\theta}_{j}\right)$. Therefore, its source power estimates histogram has a shoulder (dashed line in Fig. 4(d)) and has a larger variance. In the histogram plot, we have removed the outliers from the ML estimates. Performance results can be found in Table 1. In the table, we show the root-mean squared errors and the $\ell_{1}$ error results, which are more robust to outliers. The location error results are in terms of meters whereas the power errors are in terms of $\mathrm{dB}$.

In the second configuration shown in Fig. 4(Bottom), we localize 7 targets, where the initial locations are randomly sampled from the prior density: $p\left(\boldsymbol{\theta}_{j}\right) \sim \mathcal{N}\left(\boldsymbol{\theta}_{j \text {,true }}, 10^{2} \boldsymbol{I}_{2 \times 2}\right)$ as illustrated in Fig. 4(e). The results of the VB and ML algorithms can be found in Fig. 4(f) and (g). The histogram results are also shown in Fig. 4(h), where the ML method (dashed line) has outperformed the VB (solid line). In the histogram, the ML outliers are removed. The simulations parameters and the error results can be found in Table 1

On the average, the distribution of the location estimates in Fig. 4(b,c,f,g) show that the VB method is more susceptible to divergence whereas the ML approach is more susceptible to local minima and quite often cannot find an initial optimization direction. The divergence of the VB method can easily be determined as the large jumps are quite easy to detect whereas detecting whether the ML algorithm is stuck at a local minima is generally much harder. In addition to convergence properties, the VB method is an order of magnitude faster than the ML method in each iteration when the number of targets is 1 . As the number of targets increase, the VB method significantly outperform the ML method in computation. In most cases, we found that the VB method converges for VBIts $\leq 10$, which makes it a computationally attractive alternative even for one target as it factorizes the source location and power of the posterior.

\section{CONCLUSIONS}

In this paper, we provided a factorized VB approximation to the joint multi source energy and location posterior, which effectively decouples the estimation of the source energy and location. We compared the results with the ML approach and showed that the VB method provides computational savings while still providing competitive performance to

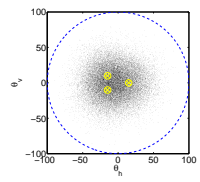

(a) Initialization

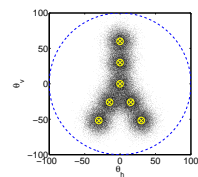

(e) Initialization

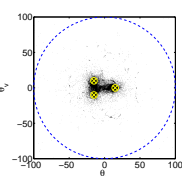

(b) VB

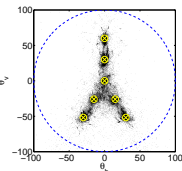

(f) $\mathrm{VB}$

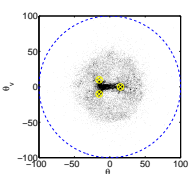

(c) $\mathrm{ML}$

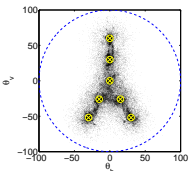

(g) $\mathrm{ML}$

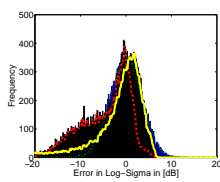

(d) VB \& ML

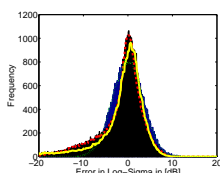

(h) VB \& ML
Fig. 4. Monte-Carlo simulation results for two multi target configurations. The sensor network boundary is also shown with dashed lines in (a-c,e-f). The true target locations are shown with $\otimes$ whereas the initial and estimated target locations are shown with dots. The histograms in (d) and (h) show the source power estimation performance (solid: VB, dashed: ML, and dotted: initialization).

the ML. For future work, we plan to extend our formulation to a distributed estimation scheme, where only a subset of sensors are used for posterior estimation to minimize the communication bandwidth among the sensors.

\section{REFERENCES}

[1] I. F. Akyildiz, W. Su, Y. Sankarasubramaniam, and E. Cayirci, "A survey on sensor networks," IEEE Communications Magazine, vol. 40, no. 8, pp. 102-114, 2002.

[2] V. Cevher, R. Chellappa, and J. H. McClellan, "Gaussian approximations for energy-based detection and localization in sensor networks," in IEEE Statistical Signal Processing Workshop, Madison, WI, 26-29 August 2007.

[3] M. I. Jordan, Z. Ghahramani, T. S. Jaakkola, and L. K. Saul, "An introduction to variational methods for graphical models," Machine Learning, vol. 37, no. 2, pp. 183-233, 1999.

[4] C. M. Bishop, Pattern recognition and machine learning, Springer, 2006.

[5] P. Morse and K. Ingard, Theoretical Acoustics, McGraw-Hill, 1968.

[6] K. L. Bell, Y. Ephraim, and H. L. Van Trees, "Explicit Ziv-Zakai lower bound for bearing estimation," IEEE Transactions on Signal Processing, vol. 44, no. 11, pp. 2810-2824, 1996.

[7] W. B. Davenport II and W. L. Root, An Introduction to the Theory of Random Signals and Noise, New York: McGraw-Hill, 1958, ch. 6, sec. 6-4, pp. 93-96.

[8] M. Evans, N. Hastings, and B. Peacock, Statistical distributions. $E d$, Wiley \& Sons. New York, 2000.

[9] L. Tierney and J. B. Kadane, "Accurate approximations for posterior moments and marginal densities," Journal of the American Statistical Association, , no. 81, pp. 82-86, 1986.

[10] P. J. Huber, "Robust estimation of a location parameter," The Annals of Mathematical Statistics, vol. 35, pp. 73-101, March 1964. 